\title{
Find and Place Sorting Technique for Unique Numbers
}

\author{
Vishweshwarayya C \\ Hallur, \\ Lecturer \\ Angadi Institute of Technology \\ \& Management, Belgaum
}

\author{
Ravindra S Hegadi, PhD. \\ Associate Professor \\ School of Computational \\ Science, \\ Solapur University, Solapur
}

\author{
S. P. Sajjan \\ Lecturer \\ Karnataka University \\ Dharwad
}

\begin{abstract}
To perform searching operation we have different kinds of searching techniques. These all searching algorithms works on data, which are previously sorted. An efficient algorithm is required to make searching fast and efficient. This paper presents a new sorting algorithm named as "Find and Place Sorting Technique for Unique Numbers (FPSTUN)". This FPSTUN is designed to perform sorting quickly and easily and also efficient as existing algorithms in sorting.
\end{abstract}

Key Words: Algorithm, Sorting, FPSTUN.

\section{INTRODUCTION}

Using a computer to solve problem involves directing it on what step it must follow to get the problem to be solved. The step it must follow is called an algorithm.

There is a direct correlation between the complexity and effectiveness of an algorithm [1].

The complexity of an algorithm is generally written in the form of $\mathrm{Big} \mathrm{O}(\mathrm{n})$ notation form, where Big $\mathrm{O}$ represents the complexity of the algorithm and value $\mathrm{n}$ represents the size of the list. The two groups of sorting algorithm are $\mathrm{O}(\mathrm{n} 2)$, which includes selection sort, shell sort, bubble sort, and insertion sort. And $\mathrm{O}(\mathrm{n} \log (\mathrm{n}))$ which includes the quick sort, heap sort, and merge sort[2].

Since the drastic advancement in computing, most of the research is done to solve the sorting problem, perhaps due to the complexity of solving it efficiently despite its simple and familiar statement. It is always very much difficult to say that one sorting technique is better than another. Performance of the various sorting algorithms depends upon the data being sorted. Sorting is very much important and is used in most of the applications and there have been plenty of performance analyses [3][4].

There has been growing interest on enhancements to sorting algorithms that do not have an effect on their asymptotic complexity but rather tend to improve performance by enhancing data locality[5].

\section{PROPOSED SYSTEM}

In FPSTUN technique the first number will be compared with all the elements in the list, at the end of each pass the selection of proper index in new list is done and then element is placed it to that position in the new list. And this step will be repeated for $\mathrm{n}$ number of times to get complete sorted list.

The best case time complexity of FPSTUN is Omega $\Omega(\mathrm{n} 2)$, the average case of the FPSTUN is theta $\Theta(\mathrm{n} 2)$ and worst case of FPSTUN is Big $\mathrm{O}(\mathrm{n} 2)$.

\section{Diagrammatic representation of ISSA:}

Unsorted List with size a[5]

\begin{tabular}{|c|c|c|c|c|}
\hline 20 & 5 & 49 & 23 & 2 \\
\hline $\mathrm{a}[0]$ & $\mathrm{a}[1]$ & $\mathrm{a}[2]$ & $\mathrm{a}[3]$ & $\mathrm{a}[4]$
\end{tabular}

Fig 1: Unsorted Array

New List i.e. b[5]

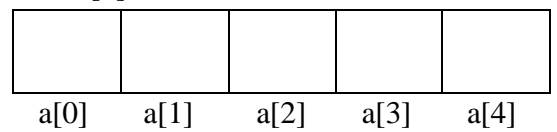

Fig 2: Empty Array

After the first pass, the index of 20 is identified and then 20 is placed in to that position in the new list. Therefore index of 20 is 2 . Hence,

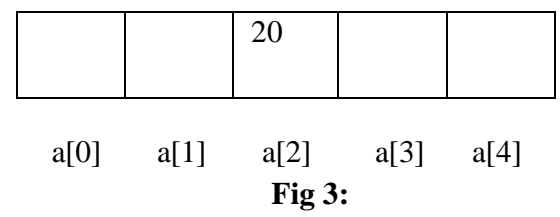

After the second pass, the index of the 5 is identified and then 5 is placed into that position in the new list. Therefore index of 5 is 1 . Hence,

\begin{tabular}{|l|l|l|l|l|}
\hline & 5 & 20 & & \\
\hline $\mathrm{a}[0]$ & $\mathrm{a}[1]$ & $\mathrm{a}[2] \quad \mathrm{a}[3]$ & $\mathrm{a}[4]$ \\
& \multicolumn{5}{c|}{ Fig 4: }
\end{tabular}

After the 3 rd pass, the index of 49 is identified and then 49 is placed into that position in new list. Therefore index of 49 is 4. Hence,

\begin{tabular}{|l|l|l|l|l|}
\hline & 5 & 20 & & 49 \\
\hline $\mathrm{a}[0]$ & $\mathrm{a}[1]$ & $\begin{array}{r}\mathrm{a}[2] \\
\text { Fig 5: }\end{array}$ & $\mathrm{a}[3]$ & $\mathrm{a}[4]$ \\
& \multicolumn{5}{|c|}{}
\end{tabular}

After the 4th pass, the index of 23 is identified and then 23 is placed into that position in the new list. Therefore index of 23 is 3 . Hence,

\begin{tabular}{ll|l|l|l|}
\hline & 5 & 20 & 23 & 49 \\
\hline $\mathrm{a}[0]$ & $\mathrm{a}[1]$ & $\mathrm{a}[2] \quad \mathrm{a}[3]$ & $\mathrm{a}[4]$ \\
& \multicolumn{5}{|c|}{ Fig 6: }
\end{tabular}


After the 5th pass, the index of 2 is calculated and then 2 is placed into that position in the new list. Therefore index of 2 is 2 . Hence,

\begin{tabular}{|l|l|l|l|l|}
\hline 2 & 5 & 20 & 23 & 49 \\
\hline \multicolumn{5}{|c|}{ a[0] } \\
\multicolumn{5}{|c|}{ Fig 7: } \\
Fin
\end{tabular}

\section{ALGORITHM}

Algorithm FPSTUN(a,b,n)

for $\mathrm{i} \leftarrow 0$ to $\mathrm{n}-1$

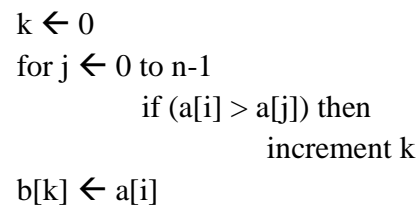

\section{COMPARISONS OF FPSTUN WITH OTHER SORTING TECHNIQUE}

Below there are some tables representing the calculated running time for $\mathrm{n}$ input values and their respective graphs in best case, average case and worst case.

a) Best Case:

Table 1. Best Case Time Complexity

\begin{tabular}{|r|r|r|r|r|}
\hline \multicolumn{5}{|c|}{ Best Case } \\
\hline & \multicolumn{1}{|c|}{ Insertion } & \multicolumn{1}{c|}{ Quick } & \multicolumn{1}{c|}{ Shell } & FPSTUN \\
\hline 10 & 10 & 10 & 10 & 100 \\
\hline 50 & 50 & 84.94 & 144.32 & 2500 \\
\hline 100 & 100 & 200 & 400 & 10000 \\
\hline 150 & 150 & 326.41 & 710.30 & 22500 \\
\hline 200 & 200 & 460.20 & 1058.94 & 40000 \\
\hline
\end{tabular}

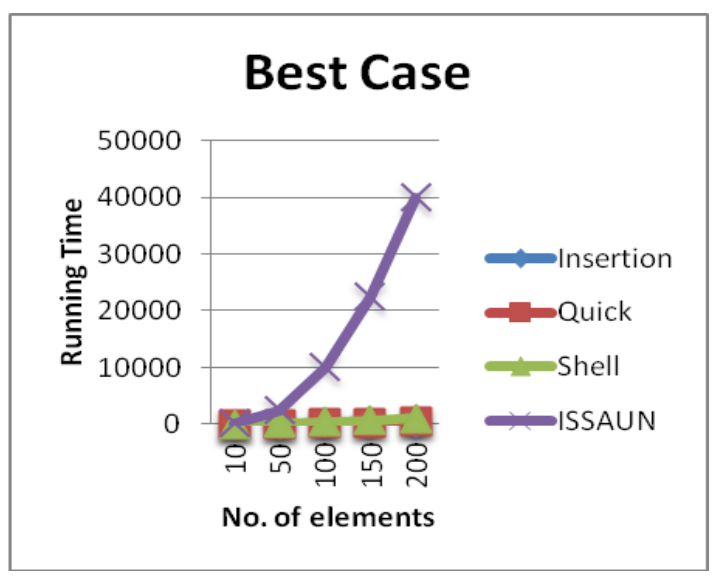

Fig 8: Best Case b) Average Case:

Table 2. Average Case Time Complexity

\begin{tabular}{|r|r|r|r|r|}
\hline \multicolumn{5}{|c|}{ Average Case } \\
\hline & Insertion & \multicolumn{1}{c|}{ Quick } & \multicolumn{1}{c|}{ Shell } & FPSTUN \\
\hline 10 & 100 & 10 & 17.78 & 100 \\
\hline 50 & 2500 & 84.94 & 132.95 & 2500 \\
\hline 100 & 10000 & 200 & 316.22 & 10000 \\
\hline 150 & 22500 & 326.41 & 524.94 & 22500 \\
\hline 200 & 40000 & 460.20 & 752.12 & 40000 \\
\hline
\end{tabular}

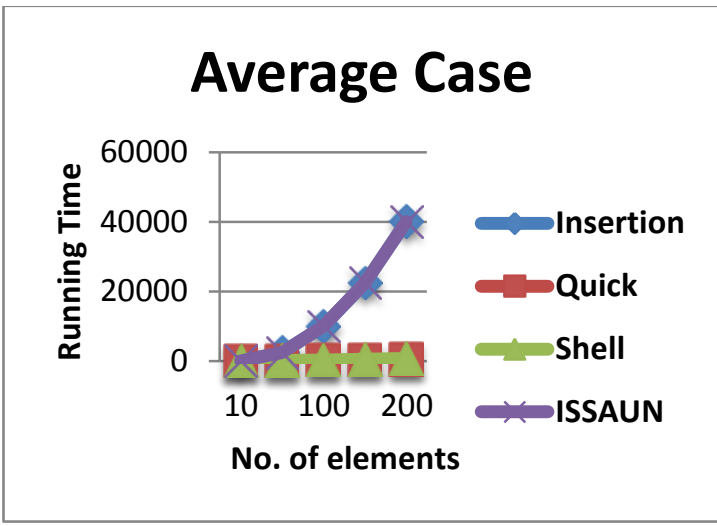

Fig 9: Average Case

c) Worst Case:

Table 3. Worst Case Time Complexity

\begin{tabular}{|r|r|r|r|r|}
\hline \multicolumn{5}{|c|}{ Worst Case } \\
\hline & Insertion & \multicolumn{1}{|c|}{ Quick } & \multicolumn{1}{c|}{ Shell } & FPSTUN \\
\hline 10 & 100 & 100 & 31.62 & 100 \\
\hline 50 & 2500 & 2500 & 353.55 & 2500 \\
\hline 100 & 10000 & 10000 & 1000 & 10000 \\
\hline 150 & 22500 & 22500 & 1837.11 & 22500 \\
\hline 200 & 40000 & 40000 & 2828.42 & 40000 \\
\hline
\end{tabular}

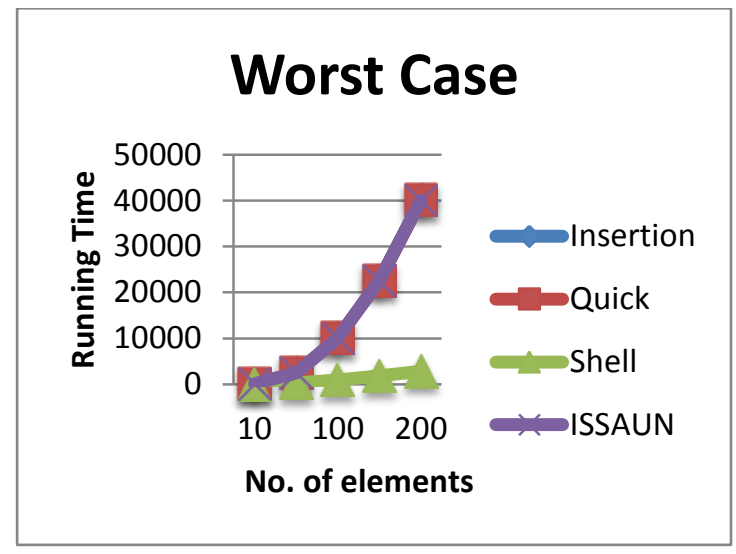

Fig 10: Worst Case 
From the above graphs for various cases complexities one can easily say that FPSTUN takes same time like selection sorting and FPSTUN is easier to implement as compare to selection sorting and in worst case it takes same time like other sorting algorithms except quick sort technique.

\section{CONCLUSION}

Logic of FPSTUN is based on the logics of well known sorting algorithms like selection sorting technique and insertion sorting technique. Hence implementation is very much easier than those two sorting techniques. And also we can easily understand the concept as we don't do any swapping in this technique. In future we will do it for duplicate numbers also.

\section{REFERENCES}

[1]. Hore, C.A.R. "Algorithm 64: Quick sort". Comm.ACM 4,7(July 1961), 321.

[2]. Soubhik Chakraborty, Mousami Bose, and Kumar Sushant, A research thesis, On way Parameters of input Distributions Need be Taken Into Account For a more precise Evalution of complexity for certain Algorithms.

[3]. J.L. Bentley and R. Sedgewick. "Fast Algorithm for Sorting and Searching Strings" ACM-SIAM SODA 97, 360-369,1997.

[4]. D.S.Malik, C++ Programming: Program Design including Data Structures, Course Technology (Thomson Learning), 2002, www.course.com

[5]. D.Jim enez-Gonz'alez, J. Navarvo, and Larriba-Pay. CCRadix: "A catch conscious sorting based on Radix sort". In Euromicro Conference on Parallel Distributed and Network based Processing. Pages 101-108, February 2003. 